\title{
Genetic algorithm optimization for pitch angle control of variable speed wind turbines
}

\author{
Mikhail Burakov*and Vladislav Shishlakov \\ State University of Aerospace Instrumentation, B. Morskaya St. 67, 190000 Saint-Petersburg, Russia
}

\begin{abstract}
In the article, the problem of optimization of the control law for rotating the blades of a wind generator with the purpose of increasing its output power is considered. The dynamics of a wind generator can be described by a nonlinear mathematical model, in which the input effect is the variable wind speed. This model is used to develop a fuzzy regulator whose parameters are tuned using a genetic algorithm. The computational experiments carried out show the practical importance of the obtained control law to ensure the effective use of the wind generator.
\end{abstract}

\section{Introduction}

Wind energy is a promising direction for the development of renewable energy sources, especially in combination with solar energy in the form of autonomous combined energy complexes [1].

Wind power plant (VEU) is a device for converting kinetic energy of wind flow into mechanical energy of rotor rotation and conversion of this energy into electric energy. The principle of operation of wind turbines: the wind flow rotates the blades and activates the rotor on which they are mounted, the rotor and blades drive the main shaft, and as a result of the generator's rotation, electricity is produced at the output [2]. According to Betz's law, a wind turbine can never have an efficiency factor greater than $59.3 \%$ [3]. In other words, the energy coming from the movement of the wind cannot be completely transformed into useful energy. The wind must move through the turbine, maintaining the optimum speed of rotation of the wind wheel. The aerodynamic power of the rotor can be adjusted by means of the angle of rotation of the blades of the wind wheel. The dynamics of the wind turbine is described by a nonlinear mathematical model, therefore it is difficult to construct an analytical dependence determining the law of the variation of the angle of inclination of the blades from the wind speed or the rotational speed of the turbine shaft.

In this paper, we consider a variant of genetic optimization of the control law for wind turbines based on experiments with its simulation model.

\section{Mathematical model of wind generator}

Consider the mathematical description of the wind turbine as a control object $[4,5]$.

The kinetic energy $E$, which the air stream has, depends on its mass and velocity can be determined by the formula:

$$
E=\frac{m v^{2}}{2}
$$

where $m$ is the mass of the air stream; $V$ - wind speed, $\mathrm{m} / \mathrm{s}$.

The air flow in the wind turbine is shown in Figure 1. The second mass air flow rate $m$ is equal to the product of the second air flow per density $\rho$ of the air flow.

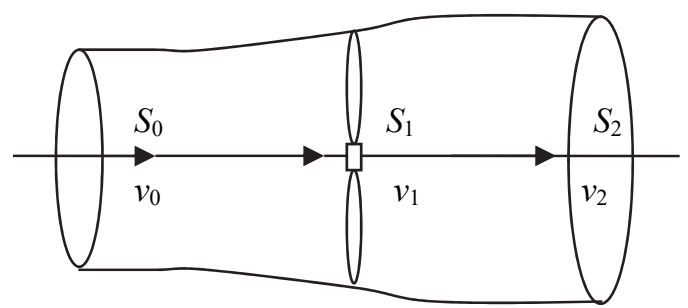

Fig. 1. Flow of air masses in a wind turbine.

According to the law of conservation of energy, the mass of the air stream of jet $m_{0}$ flowing through section $S_{0}$ is equal to the mass $m_{1}$ of this stream flowing through section $S_{1}$ and the mass of this stream flowing through section $S_{2}$, provided that the air flow is steady:

$$
\begin{gathered}
m_{0}=m_{1}=m_{2}=\text { const }, \\
m=\rho S_{0} v_{0}=\rho S_{1} v_{1}=\rho S_{2} v_{2} .
\end{gathered}
$$

Where $v_{2}<v_{1}<v_{0}, S_{1}$ is the area swept by the wind turbine, $S_{0}$ and $S_{2}$ are the cross-sectional areas of the wind flow passing through the wind turbine, respectively, to the wind wheel and behind it, $\rho$ is the air density.

This expression is called the equation of continuity of the air stream, it establishes the relationship between the jet section and wind speed.

The power of the moving air is equal to the derivative of the kinetic energy (1):

\footnotetext{
*Corresponding author: bmv@sknt.ru
} 


$$
P_{\text {wind }}=\frac{d E}{d t}=\frac{1}{2} m v=\frac{1}{2} \rho S v^{3}
$$

For the circular section, relation (2) can be written in the form:

$$
P_{\text {wind }}=\frac{1}{2} \rho \pi R^{3} v^{3}
$$

Aerodynamic power of the rotor:

$$
P=\tau_{\text {aero }} \omega,
$$

where $\tau_{\text {aero }}$ is the aerodynamic moment of rotation applied to the rotor by the wind and $\omega$ is the angular velocity of the rotor.

The power factor of the windmill (wind power factor) is defined as the ratio of the aerodynamic power of the rotor to the wind power:

$$
C_{\mathrm{p}}=\frac{P}{P_{\text {wind }}} \text {. }
$$

The power factor $C_{\mathrm{p}}(\lambda, \beta)$ is a function that depends on the two most important turbine parameters $\lambda$ and $\beta$, where $\beta$ is the rotation angle of the blades, $\lambda$ is the coefficient characterizing the screw operation or turbine speed.

Coefficient $C_{\mathrm{p}}$ - one of the main parameters characterizing the efficiency of wind turbines, it determines the average generation of electricity at a particular facility.

Another important parameter of the wind turbine is the tip speed ratio $\lambda$, defined as the ratio of the circumferential velocity of the blade ends to the effective value of wind speed:

$$
\lambda=\frac{\omega R}{v},
$$

where $R$ is the radius of the circle $(m)$, swept by the end elements of the blades; $\omega$ - angular frequency $(\mathrm{rad} / \mathrm{sec})$; $V$ - wind speed $(\mathrm{m} / \mathrm{sec})$.

The value of $\lambda$ is in the range $0.2-10$. For large wind turbines, $\lambda>1$, for wind turbines with a large number of blades $\lambda \approx 3$, for a wind turbine with three blades and a high rotational speed $\lambda \approx 6-10$.

Rotor torque:

$$
\tau_{\mathrm{c}}=k_{\mathrm{opt}} \omega^{2}
$$

where the coefficient $\mathrm{k}_{\text {opt }}[4]$ :

$$
k_{\mathrm{opt}}=\frac{1}{2} \rho S R^{3} \frac{C_{\mathrm{p}}^{\mathrm{max}}}{\lambda_{*}^{3}},
$$

where $\lambda *$ is the value of $\lambda$ in which the maximum power factor $\mathrm{Cp}$ is reached.

Angular acceleration of the rotor

$$
\dot{\omega}=\frac{1}{J}\left(\tau_{\text {aero }}-\tau_{\mathrm{c}}\right),
$$

where $J$ is the moment of inertia of the rotor, and the aerodynamic moment of rotation is described by the formula:

$$
\tau_{\text {aero }}=\frac{1}{2} \rho S R C_{\mathrm{q}}(\lambda, \beta) v^{2}
$$

where

$$
C_{\mathrm{q}}(\lambda, \beta)=\frac{C_{\mathrm{p}}(\lambda, \beta)}{\lambda} .
$$

Then

$$
\tau_{\text {aero }}=\frac{1}{2} \rho S R \frac{C_{\mathrm{p}}(\lambda, \beta)}{\lambda} v^{2},
$$

Then, substituting (6) and (8) in (7), we obtain an expression for the angular velocity of the generator

$$
\dot{\omega}=\frac{1}{2 J} \rho S R^{3} \omega^{2}\left(\frac{C_{\mathrm{p}}(\lambda, \beta)}{\lambda^{3}}-\frac{C_{\mathrm{p}}^{\max }}{\lambda_{*}^{3}}\right),
$$

In this way,

$$
\begin{aligned}
& \dot{\omega}<0, \text { if } C_{\mathrm{p}}(\lambda, \beta)<\frac{C_{p}^{\max }}{\lambda_{*}^{3}} \lambda^{3} ; \sin ^{-1} \theta \\
& \dot{\omega}>0, \text { if } C_{\mathrm{p}}(\lambda, \beta)>\frac{C_{p}^{\max }}{\lambda_{*}^{3}} \lambda^{3} .
\end{aligned}
$$

The dependence of the wind turbine power factor $\mathrm{Cp}$ on $\lambda$ and $\beta$ is expressed in the form $[4,5]$ :

$$
C_{\mathrm{p}}(\lambda, \beta)=C_{1}\left(\frac{C_{2}}{\lambda_{i}}-C_{3} \beta-C_{4}\right) e^{\frac{-C_{5}}{\lambda_{i}}}+C_{6} \lambda,
$$

Then

$$
\frac{1}{\lambda_{i}}=\frac{1}{\lambda+0.08 \beta}-\frac{0.035}{\beta^{3}+1}
$$

where the coefficients are: $C_{1}=0.5176, C_{2}=116, C_{3}=$ $0.4, C_{4}=5, C_{5}=21$ and $C_{6}=0.0068$.

Using (5), (6), (8) and (9), we can represent the dynamic model of the wind turbine in the form of the structure shown in Figure 2.

Figure 3 shows the dependence of the power factor of the turbine on the speed and the angle of inclination of the blades, found by formula (10).

As Figure 3, the maximum value of $C_{\mathrm{p}}=0.48$ is reached for $\beta=0$ and $\lambda=8.1$

\section{Principles of control system organization}

When controlling the wind turbine, the slope of the blades $\beta$ acts as a control signal (Fig. 4). A qualitative description of the operation modes of the wind turbine as a function of the wind speed is given in Figure 5. 


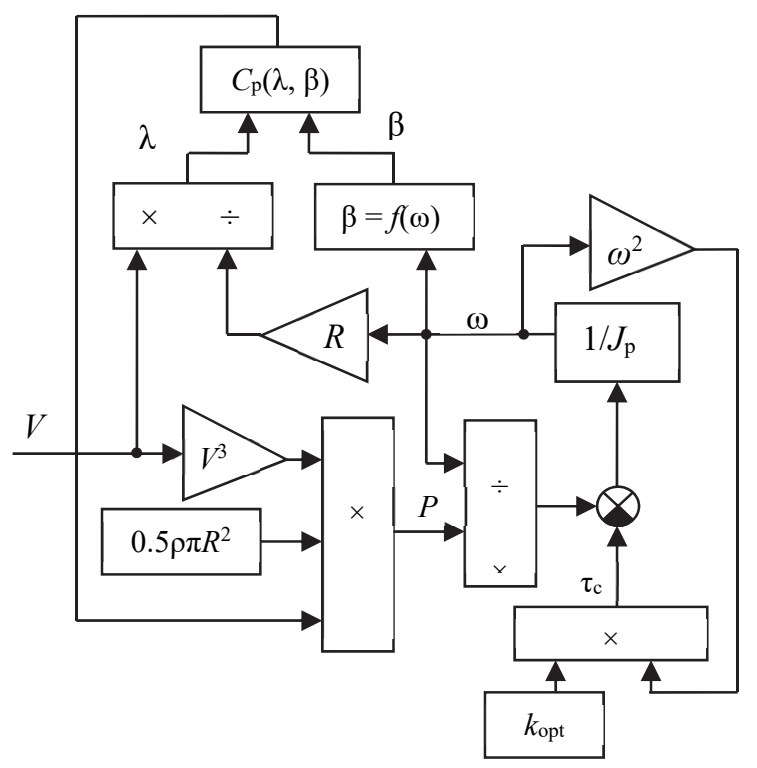

Fig. 2. Block diagram of a wind turbine as a control plant.

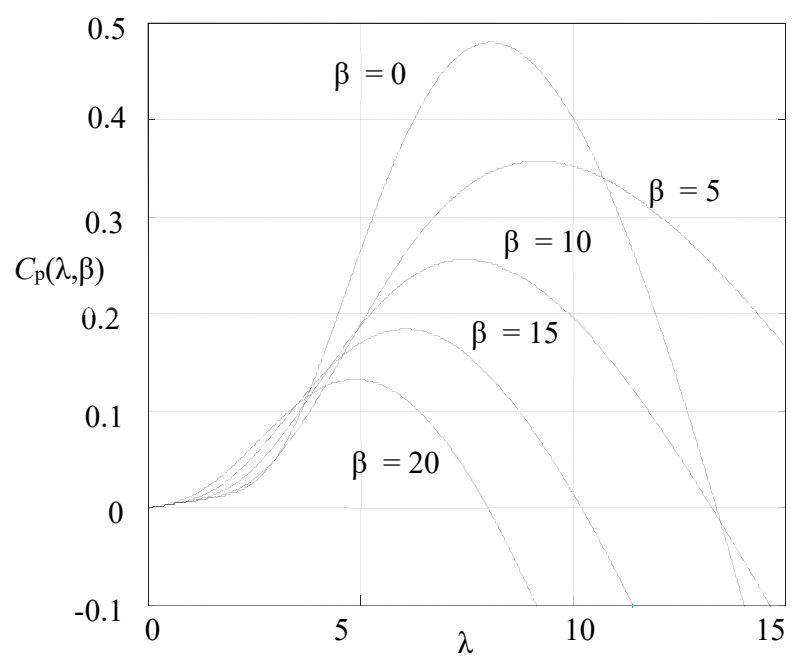

Fig. 3. Dependence of the power factor on $\lambda$ and $\beta$.

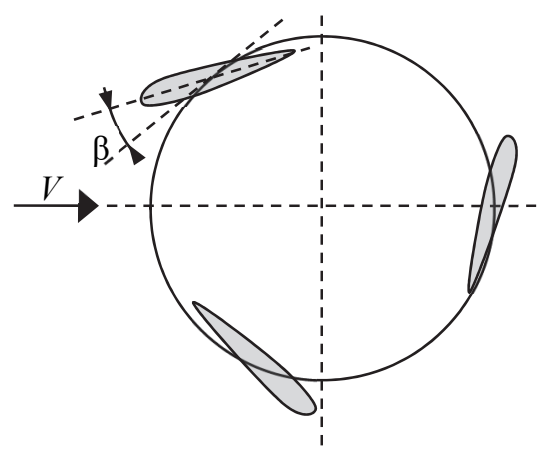

Fig. 4. Wind turbine with a vertical arrangement of blades.

Figure 5 depicts four regions:

I. Low wind speed $(0-5 \mathrm{~m} / \mathrm{sec})-$ the generator is not connected to the network.

II. The average wind speed $(5-15 \mathrm{~m} / \mathrm{sec})$, the generator is connected, but does not produce rated power.
III. High wind speed $(15-25 \mathrm{~m} / \mathrm{sec})$ - the generator is connected to the network and generates a nominal power.

IV. Critical wind speed ( $\geq 25 \mathrm{~m} / \mathrm{sec}$ ) - the generator is disconnected and the turbine is stopped.

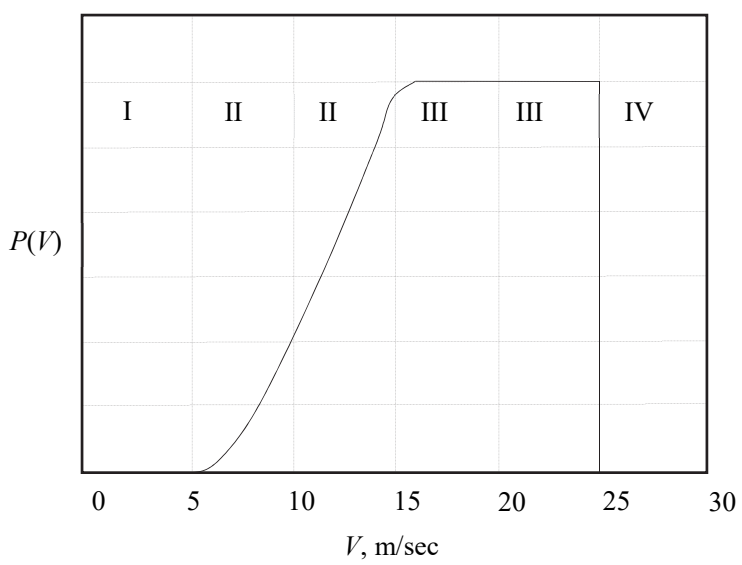

Fig. 5. Example of wind turbine operation mode.

The change in the inclination of the blades makes it possible to control the angular velocity of rotation of the turbine. The following management strategies are possible [4-6]:

1. Control of the blade inclination as a function of wind speed $\beta=f(V)$. This approach is easy to implement, but it is difficult to provide high quality, because accurate measurement of wind speed is complex (Fig. 6).

The dynamics of the servo drive is usually described by an aperiodic link:

$$
\beta=\frac{\beta^{*}}{1+T_{\mathrm{b}} s},
$$

where $T_{\mathrm{b}}$ is the servo time constant.

2. Control by the error of angular velocity (Figure 7).

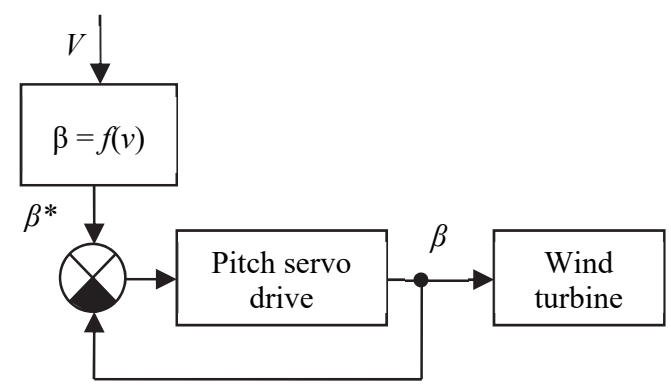

Fig. 6. Wind turbine control by wind speed.

\section{Genetic algorithm optimization of the control law}

As shown in Figure 3 and 5, as well as the above mathematical description, the control law of the wind turbine must be nonlinear, therefore, a fuzzy logic control (FLC) can be an adequate tool for organizing the control loop of the wind turbine $[7,8]$. 


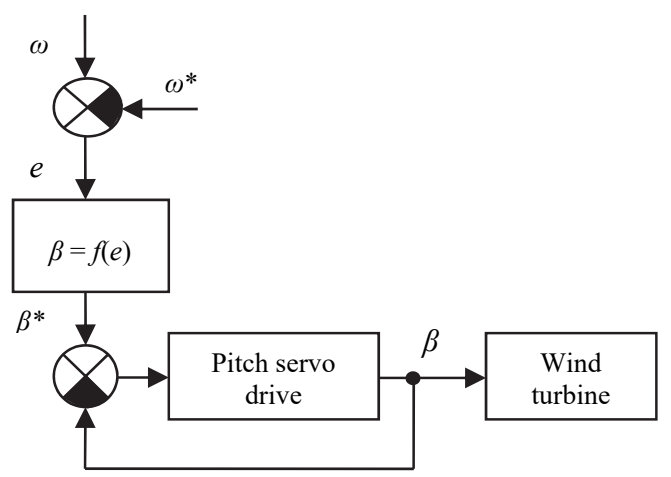

Fig. 7. Control of the wind turbine in angular velocity.

The main problem of applying FLC is the need to formalize the control law in the form of fuzzy rules that use linguistic variables to describe the inputs and outputs of the regulator.

Using triangular membership functions, it is possible to describe the control law of FLC in the form of oddsymmetric piecewise linear functions [9-10].

In accordance with Figure 6 and 7, the FLC for control of the wind turbine must realize a nonlinear static mapping: $\beta=f(V)$ or $\beta=f(\omega)$. Thus, it is possible to use P-type FLC (Fig. 8 and 9).
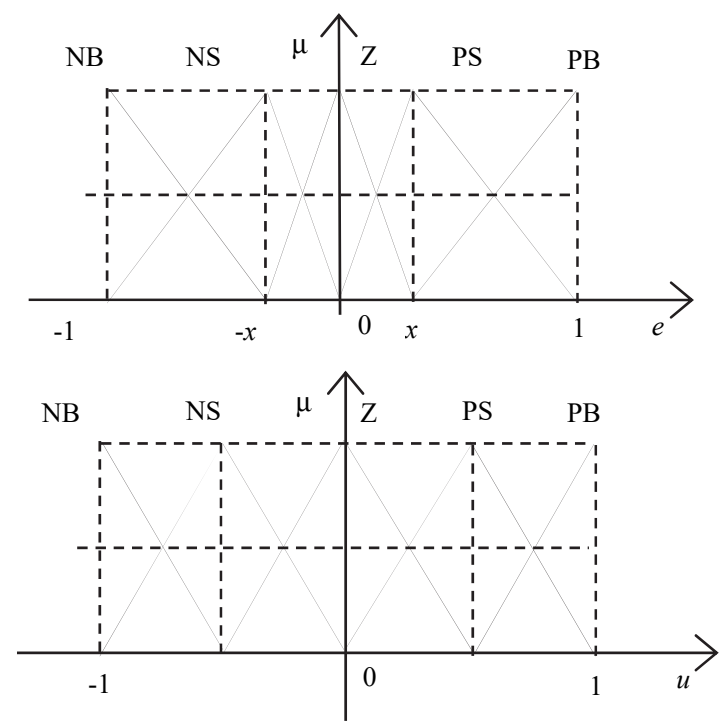

Fig. 8. Linguistic description of FLC's input and output.

When controlling the wind turbine it is impossible to heuristically describe the dependence of the inclination of the blades on the wind speed or wind speed of the wind turbine shaft. Here it is required to solve the optimization problem on the basis of experiments with the simulation model of the object. The genetic algorithm (GA) can serve as a tool for solving the problem here.

The principles of genetic adjustment of FLC are described in [9-11]. The use of GA suggests coding the parameters of the problem using chromosomes, the constituent parts of which (genes) correspond to separate parameters. The totality of chromosomes forms a population evolving in time. The goal of evolution is to improve the fitness of chromosomes, which describes the quality of the solution of the task.

The general scheme of the evolutionary optimization of FLC is shown in Figure 10.

In the problem under consideration, FLC has one input and one output, so the chromosome has a length of $2 N$, where $N$ is the number of terms used to describe wind speed. A general view of the chromosome is shown in Figure 11.

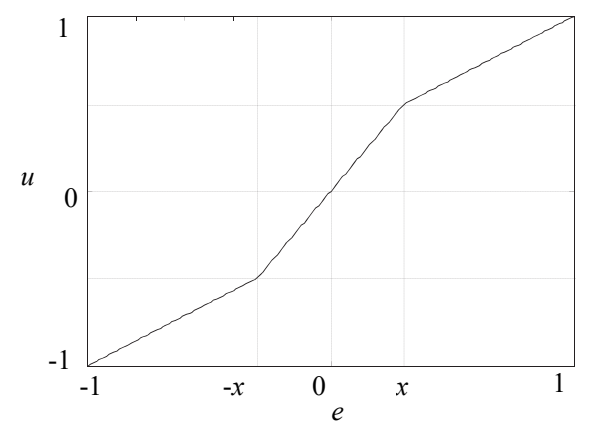

Fig. 9. Control curve of FLC.

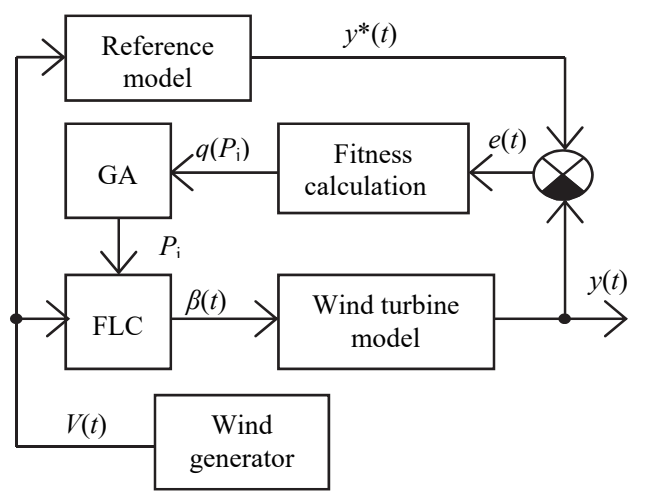

Fig. 10. Evolutionary optimization of FLC.

\begin{tabular}{|l|l|l|l|l|l|l|l|}
\hline$V_{1}$ & $V_{2}$ & $\ldots$ & $V_{\mathrm{n}}$ & $\beta_{1}$ & $\beta_{2}$ & $\ldots$ & $\beta_{\mathrm{N}}$ \\
\hline
\end{tabular}

Fig. 11. Coding parameters of FLC.

The optimization algorithm cyclically starts a wind generator that generates a test action $V(t)$. At each startup, the controller receives the parameters $P_{\mathrm{j}}$, which corresponds to the chromosome. At the end of the transient process, the parameter set receives the fitness rating $q\left(P_{\mathrm{i}}\right)$, which is formed by comparing the output of the object $y(t)$ and the reference process $y^{*}(t)$. Then the population is transformed under the influence of genetic selection, crossing and mutation operators, and new testing is taking place. The criterion for the end of the process is usually the long absence of improvements or the exhaustion of the number of given iterations.

Depending on which control option is selected wind turbine control in wind speed or wind turbine control with respect to the angular velocity - choose the option $y(t)=P(t)$ or $y(t)=\omega(t)$. Accordingly, we consider $y^{*}(t)=P_{\mathrm{R}}$ or $y^{*}(t)=\omega_{\mathrm{R}}$, where $P_{\mathrm{R}}$ and $\omega_{\mathrm{R}}$ are the rated power and rotational speed of the wind turbine. 
Then, in controlling the wind speed, the following chromosome estimate can be used:

$$
q\left(P_{i}\right)=\sum_{j=1}^{n}\left|P_{R}-P_{j}\right|,
$$

and when controlling the angular velocity, estimate:

$$
q\left(P_{i}\right)=\sum_{j=1}^{n}\left|\omega_{R}-\omega_{j}\right|,
$$

where $n$ is the number of times considered.

\section{Simulation result}

In the simulation, a wind turbine with the following parameters was considered: $R=3.1 \mathrm{~m} ; J=25.3 \mathrm{~kg} \cdot \mathrm{m} 2$; $\omega=25 \mathrm{rad} / \mathrm{sec} ; V_{\mathrm{H}}=15 \mathrm{~m} / \mathrm{sec}$. The result of genetic optimization is shown in Figure 12.

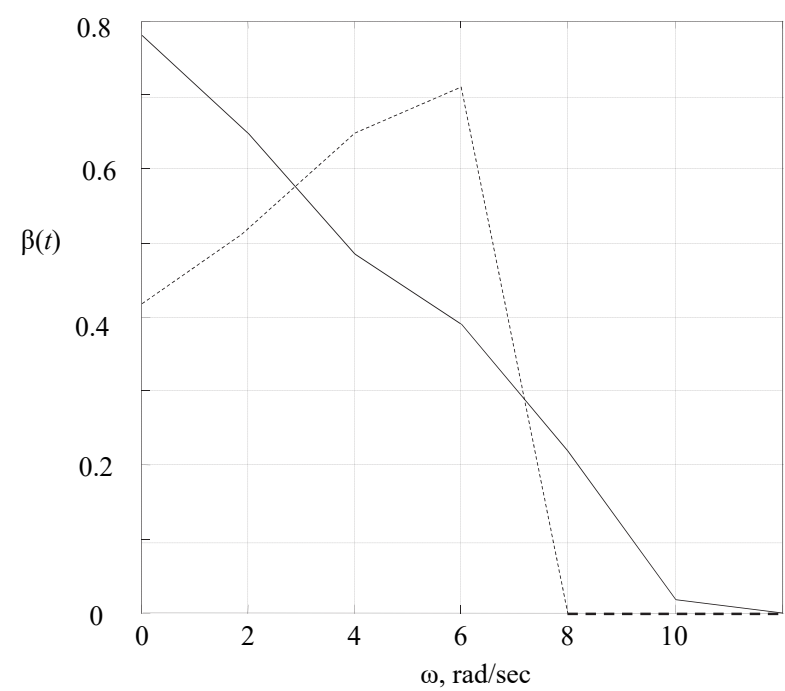

Fig. 12. The control law the inclination of the blades according to the frequency of rotation (continuous) and the wind speed (dotted line).

As Figure 12, at high wind speeds, the optimal blade angle should be close to zero. At low and medium speeds (up to $10 \mathrm{~m} / \mathrm{sec}$ ), the control law on the frequency of rotation is close to linear. The speed control is preferred due to strong fluctuations of the wind force.

Let us consider an example of a simulation of a wind turbine control system. Let the wind speed change in accordance with Figure 13.

Figure 14 shows the dynamics of output power variation during simulation. At low wind speeds, the control of the pitch of the blades has a significant effect on the output power.

Figure 15 shows the dynamics of the angular velocity of the wind wheel, Figure 16 - a change in the coefficient $C_{\mathrm{p}}$, Figure 17 - a change in the speed of the wind wheel. In the graphs, the solid line corresponds to the control of the inclination of the blades by means of FLC, and the dotted lines correspond to the constant inclination of the blades $\beta=0 \mathrm{rad}$.

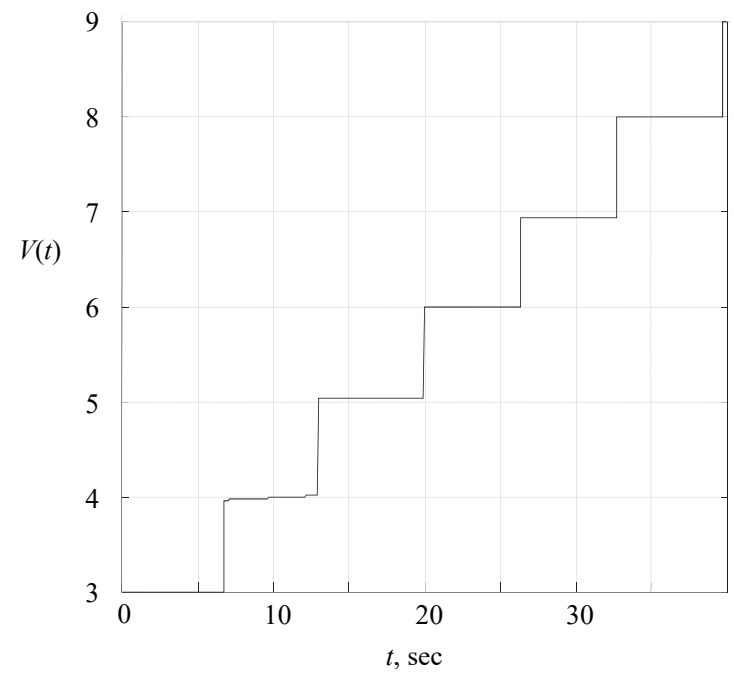

Fig. 13. Change in wind speed during simulation.

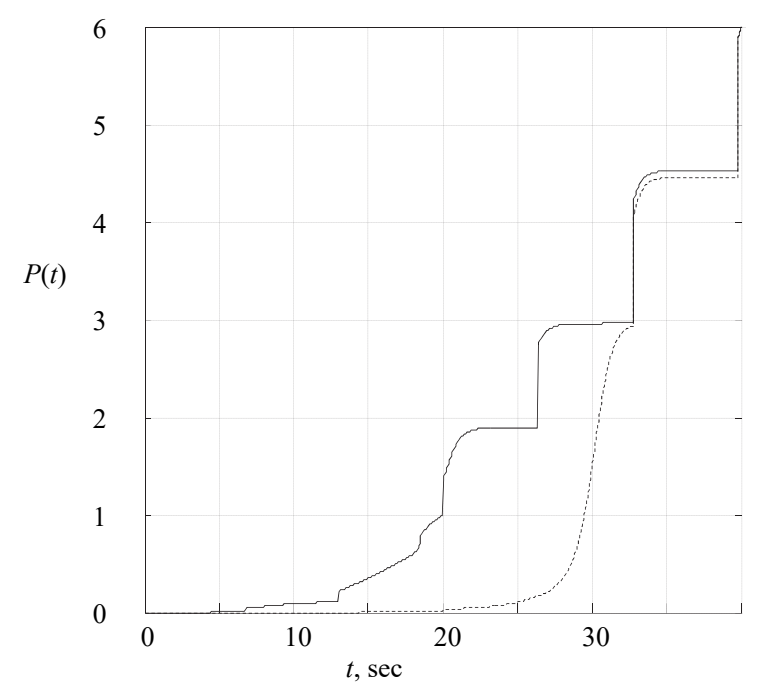

Fig. 14. Change in output power.

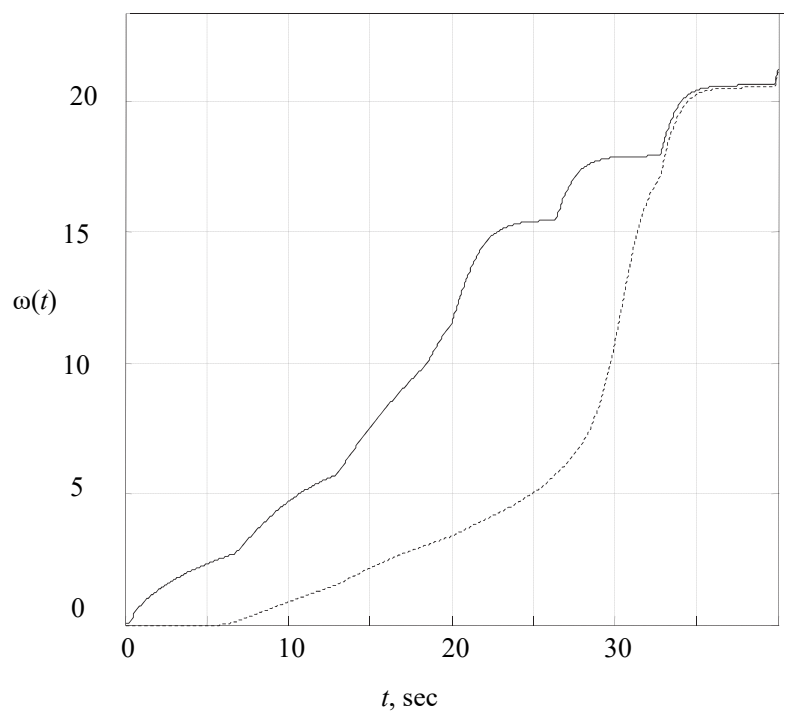

Fig. 15. Variation of the angular velocity of the wind wheel. 


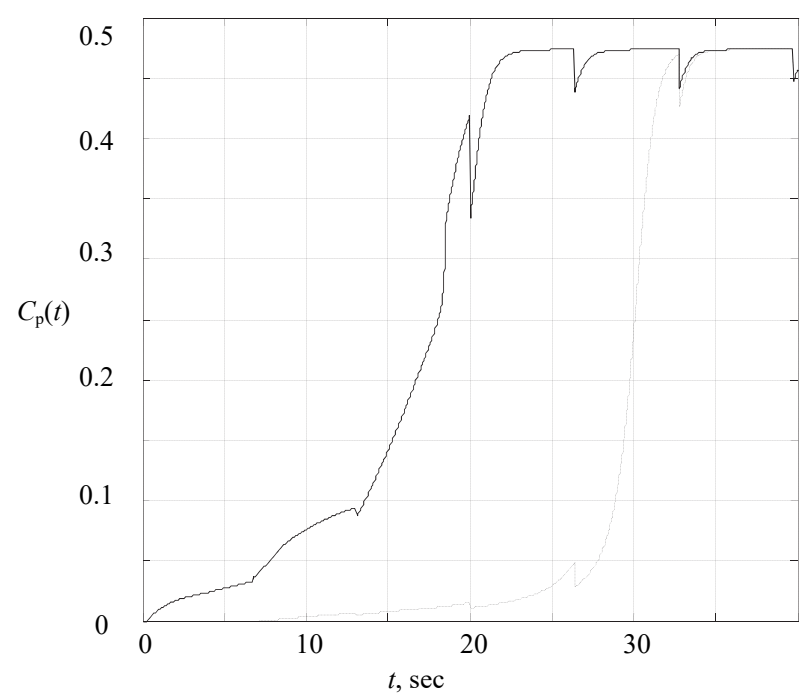

Fig. 16. Changing the power coefficient.

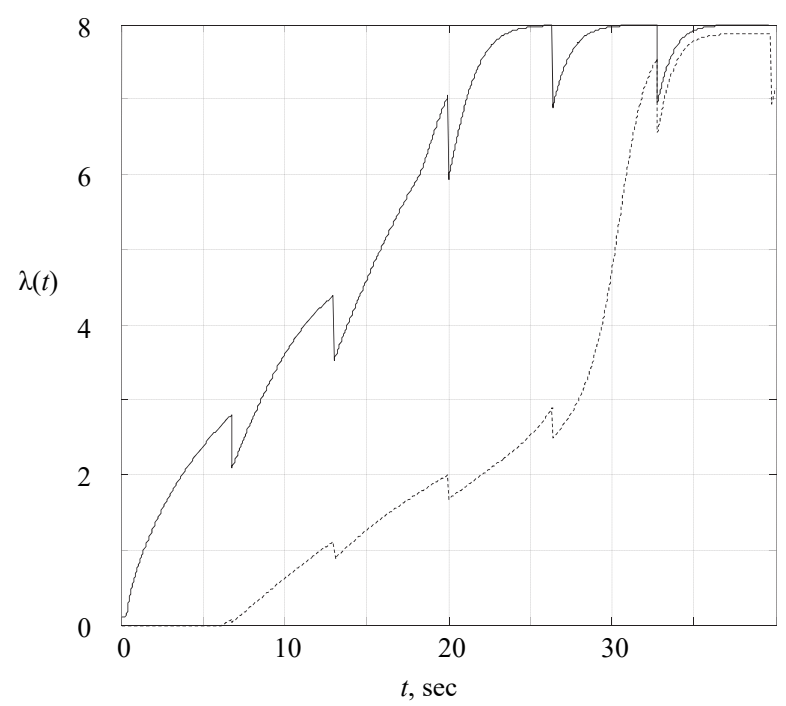

Fig. 17. Changing the tip speed ratio.

\section{Conclusion}

The approach to the synthesis of the wind turbine control law is based on the genetic optimization of the P-type FLC parameters as a result of experiments with a nonlinear computer model that takes into account all the features of the real object. The computational experiments carried out have shown that a change in the angle of inclination of the blades of the wind wheel can significantly increase the output power at low and average wind speeds. The obtained result can be useful in designing autonomous wind power plants, as well as combined renewable energy sources of low power.

\section{References}

1. L.I. Chubraeva, An.L. Ronzhin, A.V. Shyshlakov, Al.L. Ronzhin, V.F. Shyshlakov, SPIIRAS Proc. 2(33), 207-226 (2014)
2. Burton T., Sharpe D., Jenkins N., Bossanyi E., Wind Energy Handbook (John Wiley \& sons, Chichester, UK, 2001)

3. Hau E., Wind turbines: fundamentals, technologies, application, economics. (Springer Verlag, 2006)

4. Laks J., Pao L., Wright, Control of Wind Turbines: Past, Present and Future, ACC June 2009

5. L.Y. Pao, K.E. Johnson, Proc. Amer. Ctrl. Conf., June 2009

6. V. Ramakrishnan, IJESC, 1(2) (2009)

7. Passino K. M., Yurkovich S. Fuzzy control (Addison Wesley Longman, Inc. 1998)

8. O. Mengi, Turk J Elec Eng \& Comp Sci, 20(2) (2012)

9. M.V. Burakov, V.G. Kurbanov, JEAS, 11(9) (2016)

10. M.V. Burakov, V.G. Kurbanov, JEAS, 12(4) (2017)

11. M.V. Burakov, A.S. Konovalov, Kluwer International Series in Engineering and Computer Science, 664 (2002) 